\title{
A Method of Designing in Microstrip Equalizer Based on the Substructure Method
}

\author{
Huawei Zhan ${ }^{1, *}$, Haoqiang Yang ${ }^{1}$, Runan $\mathrm{Jia}^{1}$, Yu Zhang ${ }^{1}$ and Haigang Yang ${ }^{2}$ \\ ${ }^{I}$ College of Physics and Electronic Engineering, Henan Normal University, Xinxiang, China, 453007 \\ ${ }^{2}$ Renewable Energy School, North China Electric Power University, Beijing, China, 102206
}

\begin{abstract}
To make the optimization design result of system and components which, contain ordinary non-uniform substructures, meet the measurement criteria better. According to the microstrip equalizer's complex electromagnetic characteristics, the Substructure Analyzing Method of Interconnect-net based on parameters matrices measurement database is proposed and verified which combines the measure technology with database. The analysis and engineering design of the abnormal-edge microwave apparatus is realized. The method treats the complex microwave structure as a interconnect of some simpler substructure, uses the measurement database to character the substructure whose electricity and magnetism property is difficult to exactly resolve and uses the net cascading method to get the overall net parameters. The method is the basis of a number of current and pending patents. It may also lead to other fields such as the design of shortwave multimode multifeed antenna system. In this paper, a typical example of microstrip equalizer optimization is given to illustrate the efficiency of the method.
\end{abstract}

Keywords: Microstrip equalizer, method of substructure, optimization.

\section{INTRODUCTION}

Equalizer is one type of microwave passive component used widely in modern radar system, high accuracy microwave measure system and other microwave application system [1]. It can compensate various waves and irregular change of the system amplitude and phase characteristic and makes the system work in a linear transmission state.

Since 70's, the Microwave CAD, which combines microwave and computer technology, has affected the development of the microwave technology to a great degree. With the progress of software and hardware of computer, the combination of numerical method using high-speed computer technology and Electromagnetism resolution method has solved a great deal of questions of the application domain, especially the analyzing and designing of electromagnetic components. Several years ago, we brought forward the substructure analyzing method of interconnect-net [2], based on great capacity database, to settle the question that we encountered while research, for example the difficulty in analyzing and optimizing the complex electromagnetic structure, the certain blindness in designing and machining [3].

Many patents on microstrip transmission lines were invented for Equalizer [4-9]. For example, An equalizer circuit for equalizing RF frequencies was implemented as an MMIC or MIC having a series of microstrip transmission lines between an RF input terminal and an RF output terminal. It had at least one shunt path which comprised of a series combination of an FET, which was able to switch between a conducting state and a nonconducting state and a stud tuner microstrip transmission line [4]. Another equalizer circuit for RF frequencies comprised of a microstrip transmission line having a stub tuner extending transversely and having resistive means coupled to the stub tuner to de-Q the stub tune [9]. In this paper, the Substructure Analyzing Method of Interconnect - net is applied to micro-stripe equalizer's design, the theory plot the complex communication system by cascading of a set of simple substructures., The design and set up of the effective and accurate parameter measurement database of different substructure and by the use of some technologies such as transform and cascade, the micro-stripe equalizer will be designed and optimized. It is a simple, efficient and quick method. We carry out the optimization design of a kind of micro-stripe equalizer to illustrate the use of the substructure analyzing method.

\section{THE CONCEPT OF EQUILIBRIUM}

The idea of equilibrium is: if any part of signal transport system cannot transport signal without aberrance, the signal will change. To avoid this, we must design some network whos transport characteristics are complementary with the original system [10]. As a result, the whole system can transfer the signal without any change. The whole process is called equilibrium, the component or network called equalizer. Neural network techniques display more accuracy for price forecasting compared to the ARIMA models. Neural networks and SVM techniques are data driven and simple, where, they can approximate nonlinear function. These can 
be powerful and flexible tools for forecasting, if provided with enough data for training and an adequate selection.

\section{OVERVIEW OF THE SUBSTRUCTURE ANALYZ- ING METHOD OF INTERCONNECT-NET}

We define common network as: it is a kind of abstract physical model, formed with inter-connecting of network unit, converting physical quantity between their ports, so network unit is an abstract concept relative to physical part, it obeys corresponding physical laws [11]. Usually, we regard microwave net as electric net which works in microwave band. After the 1940's, microwave technology came forth as an important way of researching the electrical characteristics, since then the microwave net analyzing method has been developing rapidly. Recently, as a result of the progress of information and electronics, especially use of computers widely, microwave network is advancing more quickly than ever and many new concept, theory and method are appearing [12]. The substructure analyzing method of interconnect-net is based on the microwave engineering experience, technology of software and hardware of computer and microwave's highly precise measurement. It analyzes net using substructure idea and it's points are as follow:

1. Treat the complex net as the cascading of a set of substructures;

2. With the substructure database model, use multiple methods (which include the database, resolution and numerical method and the equivalent circuit etc.) to characterize the net property of substructures. Database is a basic method here;

3. Using the net cascading method to get the overall net parameters;

4. With the direct use of the database data and the use of some technologies such as data lookup, data interpolation, database self-completeness, data pre-compute and data visualization, the net analyzing will be performed effectively;

5. Based on the analysis, optimization design can be achieved with the use of complex optimization method and the tact-select method, process trace technology.

The original intention of this method is to solve the analysis and optimization of some complex microwave net, it can also be used in the physical net which has the similar model [13]. And compared with the existing methods, the Substructure Analyzing Method of Interconnect-net brings some new ideas:

1. It can solve the analyses and optimization of some complex but parameter measurable net;

2. It can increase the rate of analyzing and optimization of the design of some gradually changing structures greatly.

The substructure analyzing method of interconnect-net succeeded in combining microwave engineering with computer technology (database, view-data), and representing the net cell by measuring database directly. That method can analyze, optimize and design a kind of non-uniform net in engineering [14]. In this paper, we put it for optimization and design the micro-stripe equalizer.

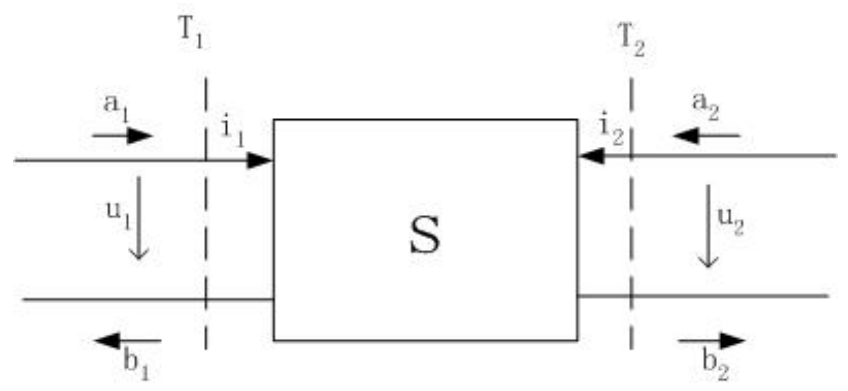

Fig. (1). The dual port s-parameter.

\section{THE FUNDAMENTAL OF SETTING OFF SUB- STRUCTURE AND CASCADE}

\subsection{Setting of Substructure}

Substructure can be basic net unit or a set of net unit due to different objects. The aim is to make analysis more quick and calculation more precise, for the convenience of debugging, designing and the optimization of the system performance [15]. The main point is which definite electronicmagnetism characteristic or net parameter be used [16].

Usually, we use the S-parameter to describe the port characteristic of a certain substructure, for it has some merits as follow:

1. Easy to measure and use.

2. S-parameter can calculate circuit with Smith chart.

3 . When the reference plane moves, the change of Sparameter is reflected on phase only.

4. It is easy to deduce the S-parameter of multiple ports network.

S-parameter of typical dual port net figure in Fig. (1). The calculating formula of S-parameter of typical dual port network is follow:

$\left\{\begin{array}{l}\mathrm{s}_{11}=\left.\frac{\mathrm{b}_{1}}{\mathrm{a}_{1}}\right|_{\mathrm{ai}_{2}=0} \text { when port } 2 \text { matching, the reflectance of port } 1 \\ \mathrm{~s}_{12}=\left.\frac{\mathrm{b}_{1}}{\mathrm{a}_{2}}\right|_{\mathrm{a}_{1}=0} \text { when port } 2 \text { matching, the reverse - } \\ \text { reflectance of port } 2 \text { to port } 1 \\ \mathrm{~s}_{21}=\left.\frac{\mathrm{b}_{2}}{\mathrm{a}_{1}}\right|_{\mathrm{a}_{2}=0} \text { when port } 1 \text { matching, the reverse - } \\ \text { reflectance of port } 1 \text { to port } 2 \\ \mathrm{~s}_{22}=\left.\frac{\mathrm{b}_{2}}{\mathrm{a}_{1}}\right|_{\mathrm{a}_{2}=0} \text { when port } 1 \text { matching, the } \mathrm{r} \\ \text { eflectance of port } 2\end{array}\right.$

\subsection{Cascade of Substructure}

It is convenient to get the parameter of overall net by using A-parameter. The calculating formula of A-parameter of typical dual port network is follow: 


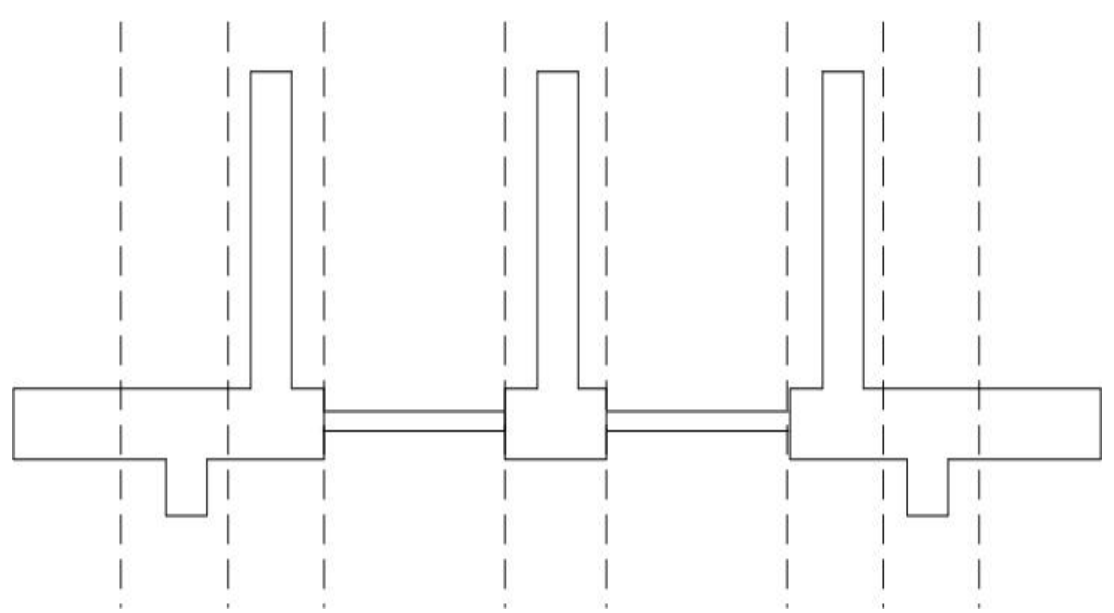

Fig. (2). The microstrip equalizer configuration.

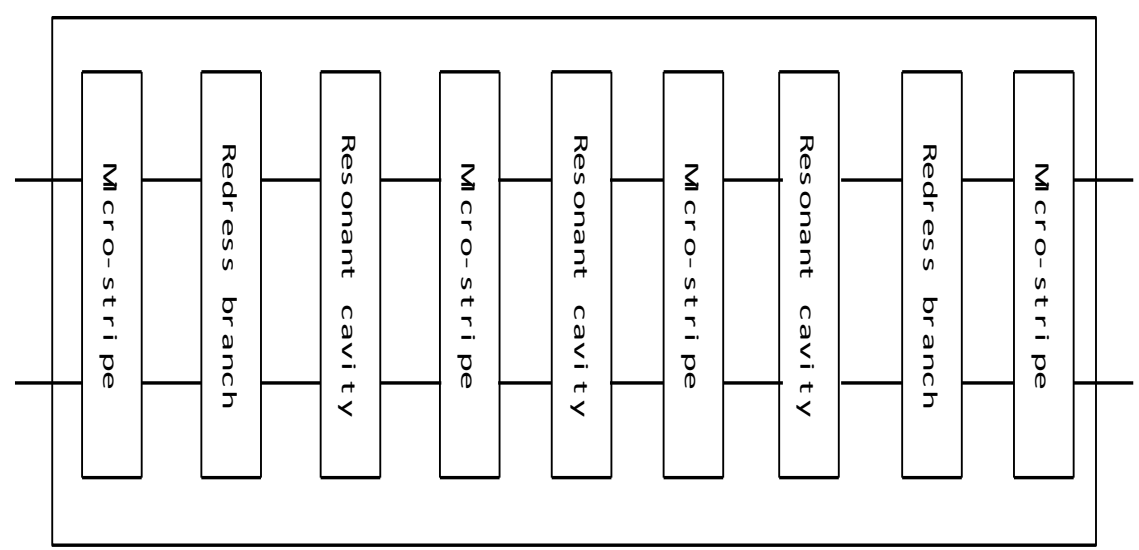

Fig. (3). The model of substructure interconnected.

$\left\{\begin{array}{l}\mathrm{a}_{11}=\left.\frac{\mathrm{u}_{1}}{\mathrm{u}_{2}}\right|_{\mathrm{i}_{2}=0} \text { output opening, the reciprocal of } \\ \text { normalize voltage transmission coefficient } \\ \mathrm{a}_{12}=\left.\frac{\mathrm{u}_{1}}{\mathrm{u}_{2}}\right|_{\mathrm{u}_{2}=0} \text { output shorting, the }\end{array}\right.$

normalize transfer admittance of input and output

$\mathrm{a}_{21}=\left.\frac{\mathrm{i}_{1}}{\mathrm{u}_{2}}\right|_{\mathrm{i}_{2}=0}$ output opening, the

(2)

normalize transfer admittance of input and output

$\mathrm{a}_{22}=-\left.\frac{\mathrm{i}_{1}}{\mathrm{i}_{2}}\right|_{\mathrm{u}_{2}=0}$ output shorting, the

reciprocal of normalize current transmission coefficient

If $\mathrm{n}$ substructures cascade, we can use formula as follow to get the overall A-parameter:

$$
[a]_{\text {all }}=[a]_{1} \bullet[a]_{2} \bullet \ldots . . \cdot[a]_{n}=\prod_{i=1}^{n}[a]_{i}
$$

\subsection{The Connection Between S-parameter and A- parameter}

During calculating, S-parameter and A-parameter can interconvert by using formula as fellow:

$$
\begin{aligned}
& \left\{\begin{array}{l}
s_{11}=\frac{a_{11}+a_{12}-a_{21}-a_{22}}{a_{11}+a_{12}+a_{21}+a_{22}} \\
s_{22}=\frac{a_{22}+a_{12}-a_{21}-a_{11}}{a_{11}+a_{12}+a_{21}+a_{22}} \\
s_{12}=\frac{2}{a_{11}+a_{12}+a_{21}+a_{22}} \\
s_{21}=\frac{2\left(a_{11} a_{22}-a_{21} a_{12}\right)}{a_{11}+a_{12}+a_{21}+a_{22}} \\
a_{11}=\frac{1}{2}\left[s_{12}+\frac{\left(1+s_{11}\right)\left(1-s_{22}\right)}{s_{21}}\right] \\
a_{12}=\frac{1}{2}\left[s_{12}-\frac{\left(1+s_{11}\right)\left(1+s_{22}\right)}{s_{21}}\right] \\
a_{21}=-\frac{1}{2}\left[s_{12}-\frac{\left(1-s_{11}\right)\left(1-s_{22}\right)}{s_{21}}\right] \\
a_{22}=\frac{1}{2}\left[s_{12}+\frac{\left(1-s_{11}\right)\left(1+s_{22}\right)}{s_{21}}\right]
\end{array}\right.
\end{aligned}
$$

\section{ANALYZING THE STRUCTURE OF MICROSTRIP EQUALIZER AND CARVING UP NET}

As to the microstrip equalizer shown in Fig. (2), a common method of constructing substructure is shown by broken lines in Fig. (3), then forming a inter-connect net which is constituted by several substructure cascades. If each sub- 


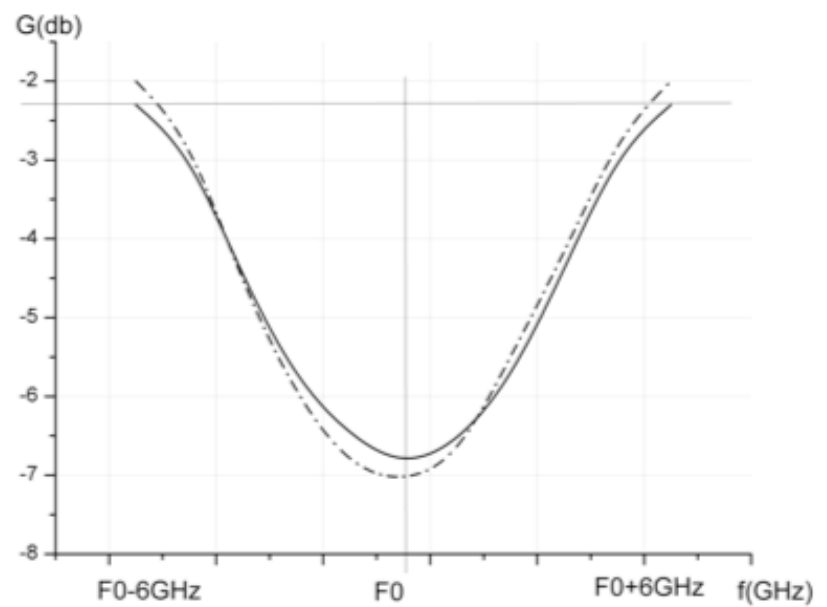

Fig. (4). The optimization result.

structure is figured by a database of S-parameter then this analyzing module is consists of several database cascades [17].

To construct database of substructure, a great deal of data is necessary. There are three ways that we can get data needed:

1. Producing some substructure components and measuring in test system to get data.

2. Using advanced EDA software (ADS, HFSS, etc) to simulate some substructure.

3. Calculating the relative simple part using resolution formula, for instance: micro-stripe.

\section{MATHEMATICAL MODEL OPTIMIZATION AND ONE TYPICAL OPTIMIZATION RESULT}

After obtaining the data representation of each substructure, we can use the method of net cascade and optimization to design the overall system. The goal is to approach target curve maximum by adjusting combined mode of various substructure [18].

We can describe certain curves by using some value of power-level, as:

$$
\begin{aligned}
& P_{i n 0}(w)=\sum_{i=0}^{n} P_{i n} \bullet \delta\left(f_{0}+\frac{B_{f}}{n} i\right) \\
& \delta(f(i))=\left\{\begin{array}{cr}
1 & i \in[0,1,2, \ldots \ldots, n] \\
0 & \text { others }
\end{array}\right.
\end{aligned}
$$

$\mathrm{B}_{\mathrm{f}}$ : bandwidth of working frequency band

$\mathrm{f}_{0}$ : floor level of working frequency band

$\mathrm{P}_{\mathrm{in}}$ : value of power-level of each frequency point

The final goal is to minimize the error between output power and the target one at the $\mathrm{N}$ frequency points. That is to say:

Set

$$
\begin{aligned}
& T(i, \mathbf{L})=P_{c} \bullet L_{A}(i) \\
& T_{0}(i)=P_{i n 0}(\omega) \\
& \mathbf{L}=\left(\mathbf{L}_{\mathrm{p}}, \mathbf{L}_{q}, \mathbf{L}_{r}, \mathbf{L}_{s}\right) \\
& =\left(L p_{1}, L p_{2}, \ldots, L p_{m}, L q_{1}, L q_{2}, \ldots, L q_{m},\right. \\
& \left.L r_{1}, L r_{2}, \ldots, L r_{m}, L s_{1}, L s_{2}, \ldots, L s_{m}\right) \\
& \text { Make } \\
& \min _{\mathbf{L}} \max _{i}\left\{\left(T(i, \mathbf{L})-T_{0}(i)\right)^{2}\right\}
\end{aligned}
$$

The standing-wave ratio pin Transmission line should satisfy the following constraint:

$$
\max _{i}\{\rho(i, \mathbf{L})\}<\rho_{0}
$$

$i$ is serial number of frequency point

When we design practically, the length, width and area of substrate and surface area of micro-stripe line can be treated as a constraint condition. All that together, we get the final mathematical model as follows:

$$
\begin{aligned}
& \min _{\mathbf{L}} \max _{i}\left\{\left(T(i, \mathbf{L})-T_{0}(i)\right)^{2}\right\} \\
& \text { s.t. }\left\{\begin{array}{l}
\max _{i}\{\rho(i, \mathbf{L})\}<\rho_{0} \\
0 \leq \mathbf{L} \mathbf{p}_{j}<5 \mathrm{~mm} \\
0 \leq \mathbf{L} q_{j} \leq 20 \mathrm{~mm} \quad i \in[0,1,2, \ldots, n] \\
0 \leq \mathbf{L} r_{j} \leq 5 \mathrm{~mm} \\
0 \leq \mathbf{L} s_{j} \leq 15 \mathrm{~mm}
\end{array}\right.
\end{aligned}
$$

This is a question with nonlinearity constraint, its augmented objective function is:

$$
\begin{aligned}
\left.F(\mathbf{L})\right|_{L=L 0} & =\min _{\mathbf{L}}\left\{\max _{i}\left\{\left[T(i, \mathbf{L})-T_{0}(i)\right]^{2}\right\}\right. \\
& +M \rho \cdot\left\{\min \left[0,\left(\rho_{0}-\max _{i} \rho(i, \mathbf{L})\right)\right]\right\}^{2} \\
& +\sum_{j=0}^{N} M_{L p j} \cdot\left\{\left[\min \left(0,5-L p_{j}\right)\right]^{2}+\left[\min \left(0, L p_{j}\right\}\right]^{2}\right\} \\
& +\sum_{j=0}^{N} M_{L q j} \cdot\left\{\left[\min \left(0,20-L q_{j}\right)\right]^{2}+\left[\min \left(0, L q_{j}\right)\right]^{2}\right\} \\
& +\sum_{j=0}^{N} M_{L r j} \cdot\left\{\left[\min \left(0,5-L r_{j}\right)\right]^{2}+\left[\min \left(0, L r_{j}\right)\right]^{2}\right\} \\
& \left.+\sum_{j=0}^{N} M_{L s j} \cdot\left\{\left[\min \left(0,15-L s_{j}\right)\right]^{2}+\left[\min \left(0, L s_{j}\right)\right]^{2}\right\}\right\} \\
& i \in[0,1, \ldots \ldots, n] \\
& j \in[1,2, \ldots \ldots, m]
\end{aligned}
$$

One typical optimization result is as shown in Fig. (4). The dotted line in the figure are the simulation results, the solid line is the best incentive curve. The simulation's results show that the optimization values are in good agreement with the metrical values. 


\section{CURRENT AND FUTURE DEVELOPMENTS}

Current and near-term future work on microstrip equalizer is described above. Usually, the measured results are more realistic than the calculation results in engineering design. By the way of reasonably measuring and establishing database of the substructure, we can avoid repeated calculation of each substructure in the process of optimization. It is effective to advance the calculation efficiency. Finally, in a more long term perspective, the fast design of complex microstrip equalizer can be realized.

\section{CONFLICT OF INTEREST}

The authors confirm that this article content has no conflict of interest.

\section{ACKNOWLEDGEMENTS}

This work is supported by National Natural Science Foundation of China (61077037), Key Scientific and Technological Project of Henan Province (102102210033), Science and Technology Research Project of The Education Department of Henan Province(14B510019), National Training Programs of Innovation and Entrepreneurship for Undergraduates, Henan Normal University (201310476099).

\section{REFERENCES}

[1] J. Kampa, and K. Petrus, "Microwave amplitude equalizer," Microwaves, Radar, and Wireless Communications, vol. 12, no. 1, pp. 37-40, Feb. 2000.

[2] H. Zhan, Z. Niu, and X. Du, "The theory research and design of feed network based on measurement database," In Proceedings of the ISTM Symposium(Conference) on Test and Measurement, Dalian, China, Jun. 2005: 7428-7431.

[3] D. Lv, D. Zhou, and Z. Wang, "Design of broadband equalizer," Journal of Microwaves, vol. 28, no. 5, pp. 93-96, May 2012.
[4] V. K. Sadhir, "Electronically tunable gain equalizer," U.S. Patent 08,042,548, Nov. 8, 1994.

[5] Z. Chen," Electrically tunable gain equalizer circuit," C.N. Patent 201,110,058,005.6, Dec. 14, 2011.

[6] Y. Jia, "Time delay equalizer for high-temperature superconduction microwave filtering group," C.N. Patent 200,510,013,239.3, Dec. 21, 2005

[7] T.-K. Chiang, "Transmission line with low dispersive properties and its application in equalization," U.S. Patent 10,927,565, Mar. 10,2005 .

[8] G. Chen, "High temperature superconductive plane type group delay equalizer for radio communication," C.N. Patent 200,510,013,388.X, Nov. 8, 2006.

[9] V. K. Sadhir, "Monolithic compatible, absorptive, amplitude shaping network," U.S. Patent 07,942,728, Feb. 1, 1994.

[10] H. Zhan, Y. Zhou, and Y. Zhang, "Property analysis of the usage in NiZn ferrite of broadband transmission-line transformer," High Power Laser and Particle Beams, vol. 22, no. 2, pp. 443-446, Feb. 2010.

[11] L. Han, and J. Shang, "A Study on microstrip equalizer with low VSWR,” Modern Radar, vol. 32, no. 5, pp. 76-78, May. 2010.

[12] A. R. Dhaini, P. H. Ho, and G. X. Shen, "Toward green nextgeneration passive optical networks," IEEE Communications Magazine, vol. 49, no. 11, pp. 94-101, Nov. 2011.

[13] Zerguine, M. K. Chan, and T. Y. Al-Naffouri, "Convergence and tracking analysis of a variable normalized LMF (XE-NLMF) algorithm," Signal Processing, vol. 89, no. 10, pp. 778-790, Dec. 2010.

[14] L. Nebuloni, and G. Orsenigo, "Microwave power module foe space applications," IEEE Transactions on Electron Devices, vol. 48, no. 1, pp. 88-94, Jan. 2001.

[15] S. V. Pato, R. Meleiro, and D. Fonseca. "All-optical burst-mode power equalizer based on cascaded SOAs for $10 \mathrm{~Gb} / \mathrm{s}$ EPONs," IEEE Photonics Technology Letters, vol. 20, no. 24, pp. 20782080, Dec. 2008.

[16] M. Pinchas, "A novel expression for the achievable mse performance obtained by blind adaptive equalizers," Signal,Image and Video Processing, vol. 7, no. 1, pp. 67-74, Jan. 2013.

[17] J. T. Yuan, and T. C. Lin "Equalization and carrier phase recovery of cma and mma in blind adaptive receivers," IEEE Transactions on Signal Processing, vol. 58, no. 6, pp. 3206-3217, Jun. 2010.

[18] L. R. Huang, W. Hong, and G. Y. Jiang, "All-optical power equalization based on a two-section reflective semiconductor optical amplifier," Optics Express, vol. 21, no. 4, pp. 4598-4611, Apr. 2013.

Received: April 11, 2014

Revised: April 28, 2014

Accepted: May 11, 2014

(C) Zhan et al.; Licensee Bentham Open.

This is an open access article licensed under the terms of the Creative Commons Attribution Non-Commercial License (http://creativecommons.org/licenses/by-nc/3.0/) which permits unrestricted, non-commercial use, distribution and reproduction in any medium, provided the work is properly cited. 